\title{
Surgical Management of Ogilvie's Syndrome Post Caesarean Section
}

\author{
Claire Chambers ${ }^{\mathrm{a}, \mathrm{b}}$, Rumina Begum ${ }^{\mathrm{a}}$, Pratik Sufi ${ }^{\mathrm{a}}$
}

\begin{abstract}
Ogilvie's syndrome or acute colonic pseudo-obstruction is a rare condition, occurring mainly in elderly people with multiple comorbidities. It is characterised by abdominal distension and dilation of the colon in the absence of a mechanical cause. Predisposing factors include infection, metabolic disturbance, cardiac disease and previous surgery. We present a case of Ogilvie's syndrome post caesarean section in a 41-year-old patient who was successfully treated with surgical management involving a temporary caecostomy. We emphasise the need for increased awareness, early diagnosis and prompt management of this syndrome to minimise the high associated morbidity and morbidity.
\end{abstract}

Keywords: Ogilvie's syndrome; Caesarean section; Acute colonic pseudo-obstruction

\section{Introduction}

In 1948 Sir Heneage Ogilvie described two cases of massive colonic dilatation without mechanical obstruction [1]. In both cases laparotomy revealed a hugely dilated atonic colon. Malignant metastases were also identified at a distant site infiltrating the region of the celiac axis and semilunar ganglion. Ogilvie hypothesised that this 'new clinical syndrome' was caused by an imbalance between sympathetic and parasympathetic innervation of the colon. We describe a case of Ogilvie's syndrome post caesarean section and discuss the pathophysiology, diagnosis and management of this condition.

\footnotetext{
Manuscript accepted for publication October 24, 2012

${ }^{a}$ Department of Surgery, The Whittington Hospital, London UK

${ }^{\mathrm{b} C o r r e s p o n d i n g ~ a u t h o r: ~ C l a i r e ~ C h a m b e r s, ~ F l a t ~ 2, ~} 45$ Maresfield

Gardens, London, NW3 5TE, UK.

Email: clairechambers1@gmail.com
}

doi: http://dx.doi.org/10.4021/jmc961w

\section{Case Report}

A healthy 41-year-old, para 2, Caucasian female was admitted at $39+4$ weeks gestation for induction of labour. Her obstetric history included previous shoulder dystocia and two spontaneous vaginal deliveries. Following an unsuccessful induction, she was transferred to labour ward for artificial rupture of membranes (ARM). In addition, syntocinon augmentation and epidural analgesia were commenced. Thirty hours post ARM, the patient underwent an emergency caesarean section as a result of poor cervical progress and the development of foetal bradycardia. A healthy male infant was born with no immediate complications.

Day 1 post caesarean section the patient complained of abdominal pain and bloating, so laxatives and peppermint water were prescribed. On Day 2 she developed abdominal distension, cough and fever. Her bowels had not opened but she was passing flatus. She was diagnosed with post-operative ileus and treated conservatively with 'drip and suck' in addition to regular laxatives and phosphate enemas.

Unfortunately, she failed to make any progress and developed vomiting and shortness of breath. On examination the abdomen was grossly distended and tympanic. Blood test revealed a leukocytosis of 20. Chest and abdomen radiographs demonstrated right lower lobe pneumonia and dilated loops of large bowel respectively (Fig. 1, 2). CT abdomen (Fig. 3) further illustrated gross right sided colonic distension, secondary small bowel dilatation, and colonic pneumatosis. A provisional diagnosis of acute colonic pseudo-obstruction was made and due to concerns of impending perforation the patient underwent an emergency laparatomy.

Laparotomy findings included dilated small bowel and colon, multiple bowel serosal tears and no evidence of perforation or mechanical obstruction. The caesarean section incision was clean and there was no bowel trauma or retained foreign bodies. An open appendicectomy was performed and the bowel was decompressed via a temporary caecostomy. The caecostomy was then closed by suturing the appendiceal stump. The serosal tears were repaired, the peritoneum washed with $7 \mathrm{~L}$ saline and clips were applied to the skin.

The patient made a good postoperative recovery but 


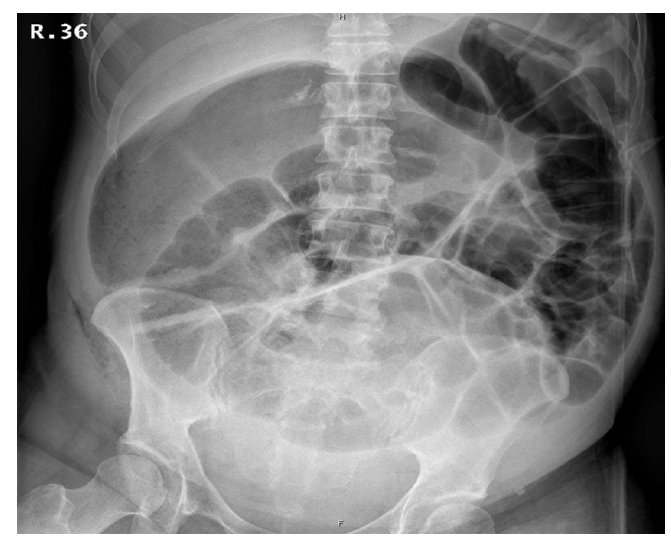

Figure 1. Abdominal X-ray shows grossly dilated bowel loops $>9 \mathrm{~cm}$.

developed a wound infection and dehiscence which settled with antibiotics and vacuum pump therapy.

\section{Discussion}

The pathophysiology of Ogilvie's syndrome is not yet clearly understood. A possible theory includes deprivation of parasympathetic supply to the colon leading to functional bowel obstruction. The successful use of neostigmine, a parasympathomimetic drug, for treatment provides support for this theory [2]. It may be that the stress of an operation or trauma results in a large increase in sympathetic drive and parasympathetic neuropraxia [3]. Ogilvie's syndrome must not be confused with neurogenic obstruction of the small intestine or paralytic ileus [4]. Multiple aetiologies have been implicated including metabolic disturbance, sepsis, spinal trauma/surgery, drugs, burns, congestive cardiac failure and post obstetric/gynaecological surgery.

The documented mortality of Ogilvie's syndrome ranges

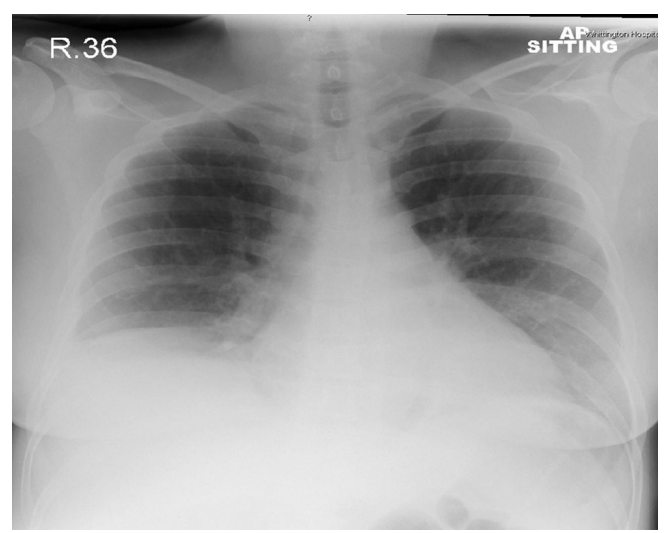

Figure 2. Chest X-ray shows loss of right heart border and splinting of the diaphragm.

from $35 \%$ to $72 \%$ [5]. It has a higher incidence in men compared to women with a ratio of $3: 2$. Despite this, caesarean section was reported as the leading cause in a large metaanalysis [6]. The association between caesarean section and Ogilvie's syndrome may be explained by the close proximity of the parasympathetic nerves (S2-S4) to the female reproductive organs, rendering them susceptible to damage intraoperatively.

As in our patient's case, the presenting symptoms include abdominal pain and distension with vomiting and hyperactive bowel sounds. Intermittent passage of stool and flatus are characteristic and should not delay investigations. Plain abdominal radiograph may show features consistent with bowel obstruction. In addition, if there are clinical features of peritonitis an erect chest X-ray should be performed to look for perforation. CT abdomen may be useful to rule out a mechanical cause for obstruction. Caecal dilation is an excellent predictor of impending bowel perforation and a caecal diameter of $9-12 \mathrm{~cm}$ is the critical point at which urgent decompression is needed to avoid bowel ischaemia

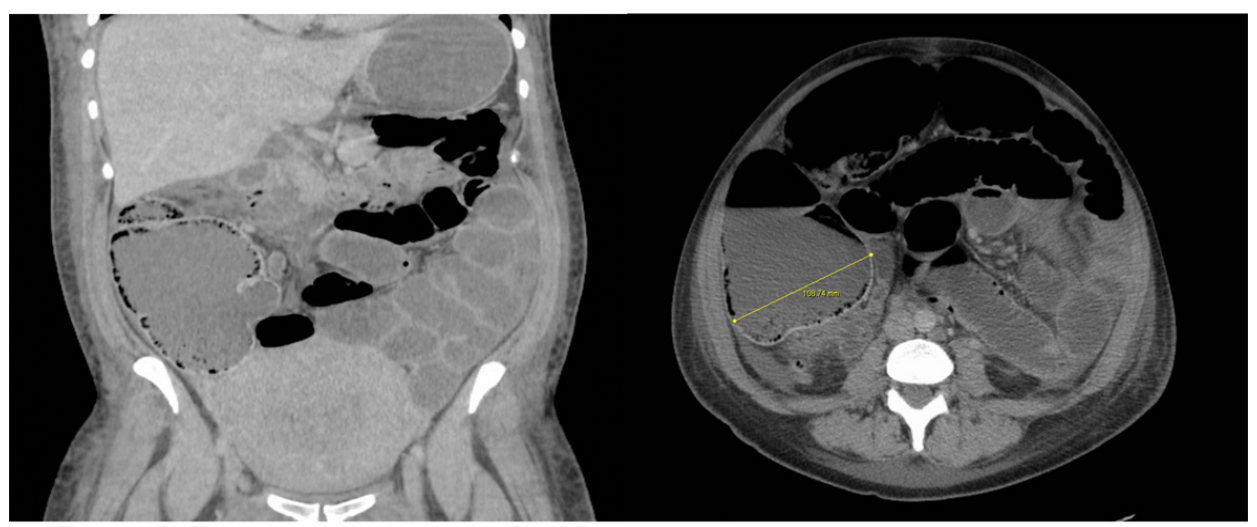

Figure 3. CT abdomen coronal and axial images showing dilated caecum, colon, small bowel and stomach. The caecal diameter is over $10 \mathrm{~cm}$. There is extensive colonic pneumatosis and no collapsed loop of bowel is shown (no evidence of mechanical obstruction). 
and perforation [7].

In the majority of patients, conservative management is successful. This involves strict fluid and electrolyte balance, intravenous fluids, and nasogastric tube insertion with routine aspiration [8]. The patient should be kept nil by mouth and all medications which may adversely affect bowel motility (such as opiates, anticholinergics and calcium channel blockers) should be discontinued. Encouraging ambulisation and mobilisation is also beneficial.

Failing conservative measures, the intravenous administration of neostigmine, an acetylcholinesterase inhibitor, has shown to be effective in $94 \%$ of patients [2]. However, neostigmine should be used cautiously with the patient kept supine with cardiac monitioring. It is contraindicated in bowel perforation or ischaemia, cardiac arrythmias, asthma and renal dysfunction.

In patients who have failed 24 - 48 hours of conservative management, colonoscopic decompression, with or without placement of a decompression tube, may be effective. Reported success rates vary between $60 \%$ and $85 \%$. However, as in our case, if there are signs of impending perforation or ischaemia then emergency laparotomy should not be delayed.

Surgical management is associated with a high mortality and morbidity. It is only implicated if there are signs of colonic ischaemia/perforation, a large caecal diameter $(>9$ $\mathrm{cm}$ ) or if conservative therapy has failed. Intraoperatively, if there is no perforated or ischaemic bowel, a caecostomy or appropriate colostomy is the procedure of choice. Otherwise a subtotal or segmental colonic resection is indicated. The bowel may then be exteriorised or an ileorectal anastomosis can be formed.

In conclusion, we have presented one of the first few cases where Ogilvie's syndrome post caesarean section has been successfully treated with surgical management involving a caecostomy performed via the appendix. Although a rare condition, it must be considered by obstetricians and general surgeons as part of the differential diagnosis in patients presenting with features of bowel obstruction. This will enable early diagnosis and rapid treatment to avoid the high related mortality and morbidity associated with this condition. A stepwise systematic approach to diagnosis and management should be implemented to ensure a successful outcome.

\section{Conflicts of Interest}

The authors declare no conflicts of interest.

\section{Grants}

No grant support has been received.

\section{References}

1. Ogilvie H. Large-intestine colic due to sympathetic deprivation; a new clinical syndrome. Br Med J. 1948;2(4579):671-673.

2. Ponec RJ, Saunders MD, Kimmey MB. Neostigmine for the treatment of acute colonic pseudo-obstruction. N Engl J Med. 1999;341(3):137-141.

3. Tenofsky PL, Beamer L, Smith RS. Ogilvie syndrome as a postoperative complication. Arch Surg. 2000;135(6):682-686; discussion 686-687.

4. Mander R, Smith GD. A systematic review of medical diagnosis of Ogilvie's syndrome in childbearing. Midwifery. 2010;26(6):573-578.

5. Dua A, Onyeka BA. Ogilvie syndrome complicated by caecal perforation in a post-caesarean section patient: a case report. Internet J Gynecol Obstet. 2006; 5.

6. Vanek VW, Al-Salti M. Acute pseudo-obstruction of the colon (Ogilvie's syndrome). An analysis of 400 cases. Dis Colon Rectum. 1986;29(3):203-210.

7. Strodel WE, Nostrant TT, Eckhauser FE, Dent TL. Therapeutic and diagnostic colonoscopy in nonobstructive colonic dilatation. Ann Surg. 1983;197(4):416-421.

8. Eisen GM, Baron TH, Dominitz JA, Faigel DO, Goldstein JL, Johanson JF, Mallery JS, et al. Acute colonic pseudoobstruction. Gastrointest Endosc. 2002;56(6):789-792. 